Gómez-Pablos, V.B., Pinto Llorente, A.M., García-Valcárcel Muñoz-Repiso, A. \& García Rodríguez, M.L. (2018). La percepción de los docentes de Bachillerato sobre un proyecto de aprendizaje-servicio. Un estudio de caso. Revista Electrónica Interuniversitaria de Formación del Profesorado, 21(2), 65-78.

\title{
La percepción de los docentes de Bachillerato sobre un proyecto de aprendizaje-servicio. Un estudio de caso
}

Verónica Basilotta Gómez-Pablos, Ana María Pinto Llorente, Ana García-Valcárcel MuñozRepiso y María Luisa García Rodríguez

Facultad de Educación, Universidad de Salamanca

\section{Resumen}

El aprendizaje y servicio es una metodología que ha tomado auge en las últimas décadas, conectada al desarrollo de competencias sociales y cívicas que despiertan el interés del alumnado por las tareas académicas. En este momento es necesario contar con investigaciones que evidencien los resultados que se obtienen con esta metodología en contextos reales de enseñanza, de modo que permitan orientar otras iniciativas y extraer conclusiones transferibles a contextos educativos. En este sentido, presentamos los resultados de un estudio de caso en el que se evalúa el proyecto "Atocha Solidaria", desarrollado en un centro educativo de Madrid, España, en el que han participado 13 docentes de Bachillerato. En este trabajo se ha analizado la opinión del profesorado que participa en el proyecto, desde un punto de vista cualitativo. Para recoger la información se ha utilizado una entrevista semiestructurada. Los resultados obtenidos muestran una valoración muy positiva por parte de los docentes, especialmente por su repercusión en el aprendizaje del alumnado, en su motivación y en el desarrollo de competencias sociales y digitales, además han destacado la implicación y participación del profesorado del centro y el apoyo del equipo directivo.

\section{Palabras clave}

Aprendizaje basado en proyectos; Aprendizaje-servicio; Profesorado; TIC.

\section{Contacto:}

Verónica Basilotta Gómez-Pablos, veronicabgp@usal.es, Facultad de Educación, Paseo de Canalejas, 169, 37008, Salamanca. 


\title{
High school teachers' perception about a service-learning project. A case study
}

\begin{abstract}
Service-learning is a methodology that has steadily grown over the last decades. It is connected to the development of social and civic competences, which arouse learners' interest in academic tasks. Nowadays, it is necessary to carry out researches that prove the results that are obtained when this methodology is implemented in real teaching contexts so that they allow guiding other initiatives and drawing conclusions that can be transferable to educational contexts. We present the results of a case study in which it is assessed the project 'Atocha Solidaria' that was developed in a high school in Madrid, Spain, and in which 13 teachers participated. The study analyses teachers' perception about the project from a qualitative perspective. The instrument chosen to collect data was a semi-structured interview. The results obtained showed that the majority of teachers had a quite positive perception of the project, specifically, participants referred to its impact in students' learning, in their motivation, and in the development of social and digital competences. Moreover, results showed teachers' involvement and participation in the project, and school management team support.
\end{abstract}

\section{Key words}

Project-Based Learning; Service-Learning; Teaching Staff; Technology.

\section{Introducción}

El aprendizaje basado en proyectos (en adelante ABP) como estrategia para el desarrollo del currículum constituye, hoy en día, una de las metodologías que más atención despierta en la escuela, como modelo que ofrece numerosas posibilidades para la formación de los alumnos. Bas (2011) y Railsback (2002) consideran que el ABP es una estrategia de instrucción auténtica y real, que promueve la resolución de problemas, la toma de decisiones, y la realización de tareas complejas y desafiantes. Asimismo, ofrece a los alumnos la posibilidad de trabajar de forma relativamente autónoma durante largos períodos de tiempo; y culmina con la elaboración de presentaciones y productos finales (García-Valcárcel y Basilotta, 2017). El ABP genera la necesidad de que estudiantes y docentes miren fuera del aula y se relacionen con el exterior. $Y$ es que el contexto social es un espacio de aprendizaje muy positivo con el cual es preciso interactuar para hacerse preguntas, recoger datos, analizarlos, interpretarlos y con todo ello, comprender la realidad e intentar mejorarla, proponiendo diversas acciones.

En este contexto, el aprendizaje-servicio (en adelante A-S) es una estrategia de trabajo que complementa el ABP a la perfección, pues fomenta que los estudiantes se planteen cómo pueden contribuir con su aprendizaje en la mejora de la comunidad y su entorno. El A-S es una propuesta educativa que consiste en poner los aprendizajes realizados en el centro educativo al servicio de la comunidad, es decir, supone un medio para promover la participación de jóvenes, adolescentes y niños en la sociedad (Escofet, Folgueiras, Luna y Palou, 2016; Vázquez, Liesa y Lozano, 2017). Los estudiantes, como personas y ciudadanos activos, participan en actividades de colaboración en las cuales movilizan sus aprendizajes para ayudar a otras personas, colectivos o instituciones. Mayor y Rodríguez (2016) 
entienden el A-S como acciones formativas orientadas a promover aprendizajes significativos, útiles y relevantes en contextos educativos ampliados (escuela-comunidad), donde el profesorado y los estudiantes conforman un grupo de investigación-acción con la intencionalidad de mejorar algunas de las necesidades sentidas en el entorno inmediato. Con el A-S, la educación en valores se pone en práctica de una manera participativa y crítica. Al mismo tiempo, es un buen instrumento para ofrecer una educación más inclusiva y desarrollar un conjunto de competencias básicas que solo pueden adquirirse a través de un trabajo global y contextualizado (Puig, Gijón, Martín y Rubio, 2011).

La utilización de las Tecnologías de la Información y la Comunicación (TIC) transforma en profundidad el desarrollo de esta metodología en dos aspectos fundamentales: a) el acceso y la gestión de la información, b) la comunicación del estudiante con el profesor, sus compañeros y la comunidad (Badía y García, 2006). Los estudiantes pueden usar las tecnologías para acceder a datos reales en la web, recopilar y analizar datos, interactuar y colaborar con otros, crear modelos y producir artefactos multimedia. Además no solo contribuye aumentando el interés del alumno por el proyecto, sino que ayuda al profesor de forma indirecta durante el proceso, ofreciendo apoyo a los estudiantes a medida que recopilan información y la utilizan para generar productos reales y auténticos que reflejen su aprendizaje (Barak y Dori, 2004)

Realizar en la escuela proyectos de A-S significa abrirse a la sociedad, crear redes entre instituciones y establecer contextos de aprendizaje en los que se ponen en práctica habilidades comunicativas capaces de regular la participación en debates, en proyectos solidarios en los que los participantes se impliquen formando parte e interviniendo en ellos (Cámara, Díaz y Ortega, 2017).

Este motivo y también, el auge que ha tomado esta forma de enfocar el ABP en la escuela, justifican el hecho de llevar a cabo el estudio de caso de un proyecto educativo de A-S, pues consideramos que tiene un alto poder formativo, ya que combina en una sola actividad el aprendizaje de contenidos, competencias y valores, con la realización de tareas al servicio de la comunidad.

\section{El proyecto Atocha Solidaria}

El objetivo del proyecto Atocha Solidaria (http://atochasolidaria.orgl) es dar visibilidad a algunas ONG en su labor solidaria con los más desfavorecidos de su entorno. Para conseguir este objetivo, el proyecto se ha estructurado en varias fases y ha seguido el método de Design for Change, un movimiento internacional, que surge en la India en 2009 cuyo objetivo es ofrecer a niños y jóvenes la oportunidad de poner en práctica sus propias ideas para cambiar el mundo desde su propio entorno. Se basa en el proceso de Design Thinking, un método de resolución de retos profundamente humano cuyos pilares fundamentales son la creatividad, el pensamiento lógico, la colaboración, la empatía y el aprendizaje del error.

En este proyecto, los alumnos se organizaron en equipos de trabajo de 405 integrantes y cada uno asumió un rol: guía, portavoz, community manager, cronista y fotógrafo. Los roles se iban rotando entre los integrantes de cada equipo, a excepción del guía, el cual fue designado por el profesor y realizado siempre por el mismo alumno. También cada grupo disponía de un profesor mentor que le asesoraba y ayudaba durante todo el proceso.

El proyecto se ha organizado en tres fases diferenciadas: inmersión, desarrollo y difusión. En la primera fase, que se desarrolló durante una semana, los estudiantes llevaron a cabo un proceso de inmersión en las diferentes ONG, participantes en el proyecto, para conocer a fondo el trabajo que realizaban, así como sus labores sociales. Esta fase del proyecto se 
ha desarrollado siguiendo las etapas del proceso de Design for change: siente, imagina, actúa, evolúa y comparte.

- Siente: es la etapa en la que los alumnos identifican posibles focos de acción a partir de situaciones de su entorno que les gustaría que se diesen de otra manera. En esta fase los alumnos tuvieron que seleccionar una ONG de un distrito de Madrid, y buscar información en Internet sobre ella. Además pensaron en un reto que pudiese mejorar la actuación de su ONG. Por ejemplo, aumentar su visibilidad en las redes sociales, conseguir fondos económicos, etc.

- Imagina: comprende la generación y el desarrollo de las ideas para mejorar las situaciones analizadas en la etapa anterior y la preparación para ponerlas en práctica. En esta fase, los alumnos propusieron ideas para trabajar el reto que habían seleccionado en la fase anterior. Por ejemplo, crear una cuenta en Instagram para difundir la actividad de la ONG, realizar un bocadillo solidario para recaudar fondos económicos, etc.

- Actúa: es el paso a la acción, el momento en que sus propuestas de cambio se llevan a la realidad. En esta fase, los estudiantes comenzaron a poner en práctica sus retos, contactaron con sus ONG, se entrevistaron con ellas, y les invitaron a colaborar.

- Evolúa: este término responde a un juego de palabras que incluye el concepto de evolución y evaluación. Los estudiantes reflexionan sobre la experiencia vivida y se imaginan posibles acciones futuras. Esta fase se ha desarrollado a lo largo de todo el proyecto; los estudiantes, en grupos de trabajo, han cumplimentado cada día una escalera de metacognición para valorar las actividades realizadas y han confeccionado un blog como registro de su trabajo.

- Comparte: es el momento en que los alumnos hacen visibles sus proyectos y sus vivencias a otras personas, pensando qué ha funcionado bien y qué se podría mejorar. Al igual que la fase anterior, esta se ha desarrollado durante todo el proyecto. Los estudiantes han compartido en redes sociales fotos y comentarios del proceso.

En la etapa de desarrollo, que duró un mes y medio, los estudiantes pusieron en marcha sus retos para mejorar la acción de las organizaciones seleccionadas. Al final de la misma, y durante dos días, presentaron sus ideas ante estas ONG en el espacio MediaLab del CaixaForum, en Madrid (España) en estrecha colaboración con los estudiantes de Formación Profesional del colegio, que les ayudaron a elaborar diferentes carteles para la exposición. Además los estudiantes de Bachillerato realizaron diversos talleres con alumnos de Primaria para mostrarles la labor de sus ONG.

Tanto los estudiantes como los docentes han utilizado herramientas de la web 2.0, principalmente Twitter y Blogger; y aplicaciones para móviles y tablets con las que manejar la información en diferentes formatos (vídeos, audio, presentaciones, texto,... ) y favorecer el proceso de aprendizaje.

\section{Metodología}

\section{Objetivo del estudio}

El objetivo principal de este estudio es analizar los aspectos más satisfactorios del proyecto Atocha Solidaria desde el punto de vista de los docentes. En concreto, la pregunta de 
investigación mediante la cual se quiere dar respuesta a este objetivo es: ¿qué aspectos positivos del proyecto destaca el profesorado?

Consideramos que este análisis servirá para orientar a otros docentes que opten por este tipo de iniciativas para abordar el currículum escolar con nuevas perspectivas, y permitirá extraer conclusiones transferibles a otros contextos educativos interesados en la renovación metodológica y el aprendizaje basado en proyectos.

\section{Diseño de la investigación}

Esta investigación forma parte de un estudio de caso en la línea de lo que Stenhouse (1991) denomina "estudio educativo de casos": investigaciones cuyo propósito es mejorar la práctica educativa (Lacueva, Imbernón y Llobera, 2003). El estudio de casos es una investigación exhaustiva, cuya finalidad primordial es generar una comprensión profunda de un tema determinado, un programa, una política, una institución o un sistema para generar conocimientos y/o informar el desarrollo de políticas, la práctica profesional y la acción civil o de la comunidad.

En esta investigación se ha definido el caso como un proyecto educativo, un proyecto en el que se trabaja de forma colaborativa y se utilizan herramientas tecnológicas para llevarlo a cabo. Dada la naturaleza del caso podríamos denominarlo ejemplar, porque es presentado como un ejemplo ilustrativo de experiencia educativa innovadora. Si consideramos el tipo de acontecimiento que se analiza, podemos hablar de un caso sincrónico o contemporáneo porque el análisis del proyecto tiene lugar en el momento en que se desarrolla la investigación (García-Valcárcel, 2015).

\section{Participantes}

En este proyecto participaron 13 docentes de un centro educativo de Madrid, que se distribuyen de la siguiente manera: 6 hombres (46\%) y 7 mujeres (54\%), los cuales presentan edades comprendidas entre 21 y 60 años. En general, este profesorado cuenta con una larga y extensa trayectoria docente, concretamente cinco de ellos llevan más de quince años dando clases en el centro educativo. Una de ellas es la coordinadora del proyecto, Charo Fernández (http://yalocin.com/) profesora de informática y presidenta de la asociación Aulablog (http://www.aulablog.com/blog/), una comunidad de profesores de diferentes puntos de España interesados en promover el uso de las TIC en la educación, especialmente de todas las herramientas enmarcadas en lo que se conoce como Web Educativa 2.0.

\section{Instrumento}

Para recoger la información, se realiza una entrevista semiestructurada con cada docente que ha participado en el proyecto, con el objetivo de realizar una valoración global del mismo, recopilar datos sobre el contexto, la metodología, las competencias profesionales puestas en juego y la evaluación de los resultados. La entrevista se orientó en base a un guion semi-estructurado previamente establecido y en directa relación con la pregunta de investigación señalada al inicio de este trabajo. Aun así el guion adoptado se planteó como un instrumento flexible que, en diversas ocasiones, se ha ido adecuando a la realidad contextual en el que se administraba lo que ha permitido explorar ciertos matices inicialmente no recogidos.

La información sobre el caso se ha recogido durante el curso académico 2015-2016, gracias a la colaboración de los docentes implicados. Ellos han permitido el acceso al aula para observar la dinámica del trabajo con la que se desarrolla el proyecto y han atendido las demandas de la entrevista llevada a cabo para la recogida de información al finalizar el 
mismo. Todas las entrevistas han sido grabadas en audio y posteriormente transcritas, previo permiso de cada uno de los participantes de forma individualizada.

\section{Análisis de datos}

El análisis del contenido de las entrevistas se ha focalizado en los aspectos positivos que los docentes perciben en el proyecto, considerando que estas concepciones son las que determinan en gran medida el éxito del mismo. Así pues, se ha extraído el contenido de las entrevistas relacionadas con este tópico y de manera inductiva se ha elaborado el sistema de categorías, el cual ha sido validado por cinco expertos, y se ha sometido a control a través de la doble categorización de siete entrevistas (entrevistas impares) por parte de dos investigadores diferentes, obteniendo un alto índice de fiabilidad al observarse una alta concordancia (Kappa de Cohen > 90) (Tabla 1). Para el análisis del contenido de las entrevistas se ha utilizado el programa NVivo11, que ha permitido obtener las nubes de palabras y las frecuencias de las categorías.

Tabla 1.

Índice Kappa de Cohen en entrevistas impares

\begin{tabular}{|l|l|l|l|}
\hline Entrevista & Kappa & Acuerdo (\%) & Desacuerdo (\%) \\
\hline 1 & 0,95 & 98,72 & 1,28 \\
\hline 3 & 0,93 & 98,78 & 1,22 \\
\hline 5 & 0,97 & 99,37 & 0,63 \\
\hline 7 & 0,96 & 98,57 & 1,43 \\
\hline 9 & 0,95 & 98,73 & 1,27 \\
\hline 11 & 0,98 & 99,35 & 0,65 \\
\hline 13 & 0,95 & 98,04 & 1,96 \\
\hline
\end{tabular}

\section{Resultados}

Obtenemos en primer lugar una nube de palabras con el programa Nvivo11 con el objetivo de explorar qué términos aparecen con más frecuencia en el discurso de los docentes cuando mencionan aspectos positivos. De este modo, se solicita al programa la obtención de una nube de 30 palabras con una longitud mínima de tres letras. Habitualmente aparecen algunas palabras frecuentes que tienen poco contenido referencial, por lo que no son indicativas de las representaciones de un texto. Beaugrande y Dressler (1997) distinguen entre las palabras funcionales y las palabras con contenido que son más informativas. Una estrategia que se ha empleado para evitar la aparición de estas palabras es crear una lista de exclusión de palabras que no son consideradas en los análisis de frecuencias. Tal y como se muestra en la Figura 1 las palabras que aparecen con mayor frecuencia son: proyecto (60 referencias), hacer ( 51 referencias), bien ( 47 referencias) trabajo (44 referencias), chicos ( 43 referencias) y ONG (39 referencias). 


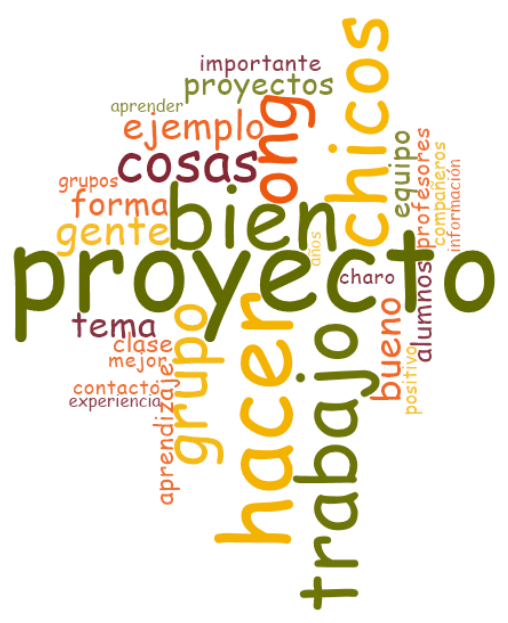

Figura 1. Nube de palabras de aspectos positivos del proyecto desde la perspectiva del profesorado

A continuación hemos escogido la palabra clave "ONG" para analizarla en su contexto, obteniendo el árbol de palabras y frases que se muestra en la figura 2. En este árbol se recogen las frases de las diferentes entrevistas en las que aparece el término seleccionado y el número entre paréntesis identifica la entrevista (García-Valcárcel, Basilotta y López, 2014).

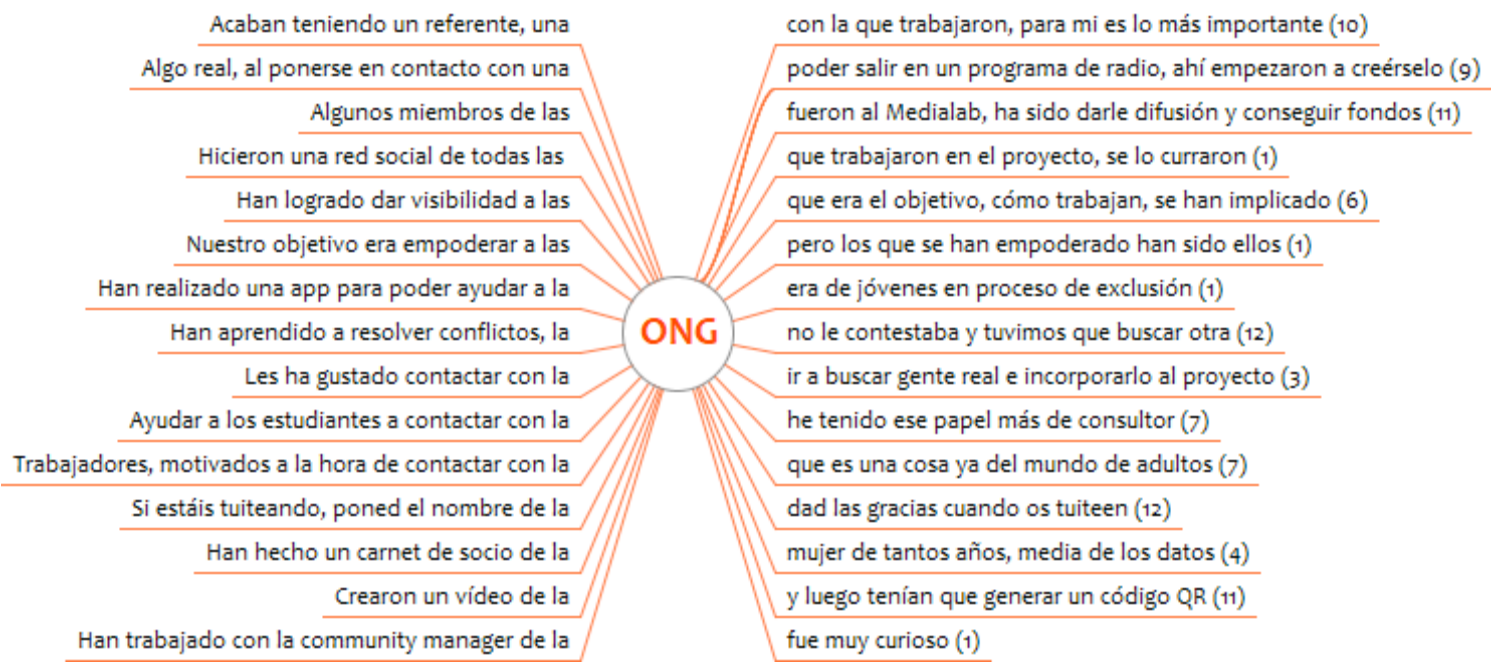

Figura 2. Árbol de palabras y frases de la palabra clave "ONG"

Se pueden resaltar del árbol algunas ideas que nos parecen de especial interés en el análisis del tema y que resumen de alguna manera lo expresado por este grupo de docentes en relación al potencial del proyecto en el aprendizaje y servicio: "Algo real, ponerse en contacto con una ONG, poder salir en un programa de radio"; "Han logrado dar visibilidad a las ONG que era el objetivo, cómo trabajan, se han implicado"; "Nuestro objetivo era empoderar a las ONG, pero los que se han empoderado han sido ellos"; "Han realizado una app para poder ayudar a la ONG, era de jóvenes en proceso de exclusión"; "Han aprendido a resolver conflictos, la ONG no le contestaba y tuvimos que buscar otra"; "Ayudar a los estudiantes a contactar con la ONG, he tenido ese papel más de consultor"; "Han trabajado con la community manager de la ONG, ha sido muy curioso". 
Una vez analizada la figura 2, obtenemos el número y porcentaje de referencias textuales de cada una de las categorías que se refieren a los aspectos positivos que los docentes atribuyen al proyecto Atocha Solidaria. Para argumentar su sensación, el profesorado menciona logros y datos que afectan a toda la comunidad educativa tal y como podemos observar en la Tabla 2, en la que se muestran las frecuencias de cada una de las categorías que hemos identificado.

Tabla 2.

Aspectos positivos del proyecto desde la perspectiva del profesorado. Sistema de categorías

\begin{tabular}{|l|l|l|}
\hline Sistema de categorías & $\begin{array}{l}\text { Referencias } \\
\text { textuales }\end{array}$ & $\%$ \\
\hline Aspectos positivos del proyecto Atocha Solidaria & 363 & 100 \\
\hline 1. Respecto al alumnado & 190 & 52,34 \\
\hline 1.1. Adquisición de competencias clave & 124 & 34,15 \\
\hline 1.1.1. Competencia aprender a aprender & 12 & 3,30 \\
\hline 1.1.2. Competencia comunicativa lingüística & 10 & 2,75 \\
\hline $\begin{array}{l}\text { 1.1.3. Competencia de conciencia y expresión } \\
\text { cultural }\end{array}$ & 6 & 1,65 \\
\hline $\begin{array}{l}\text { 1.1.4. Competencia del sentido de la iniciativa y } \\
\text { espíritu emprendedor }\end{array}$ & 23 & 6,33 \\
\hline 1.1.5. Competencia digital & 24 & 6,61 \\
\hline $\begin{array}{l}\text { 1.1.6. Competencia matemática y } \\
\text { competencias básicas en ciencia y tecnología }\end{array}$ & 4 & 1,10 \\
\hline 1.1.7. Competencias sociales y cívicas & 44 & 12,12 \\
\hline \begin{tabular}{l} 
1.1.7.1. Desarrollo afectivo y emocional \\
\hline 1.1.7.2. Educación ciudadana
\end{tabular} & 4 & 1,10 \\
\hline $\begin{array}{l}\text { 1.1.7.3. Interacción y colaboración } \\
\text { 1.2. Aprendizaje aplicado }\end{array}$ & 8 & 2,20 \\
\hline 1.3. Implicación y participación & 6,78 \\
\hline 1.4. Motivación & 22 & 6,06 \\
\hline 1.5. Protagonismo del alumnado de problemas & 8,65 \\
\hline
\end{tabular}




\begin{tabular}{|c|c|c|}
\hline Sistema de categorías & $\begin{array}{l}\text { Referencias } \\
\text { textuales }\end{array}$ & $\%$ \\
\hline 1.6. Satisfacción personal & 6 & 1,65 \\
\hline 2. Respecto al profesorado & 127 & 34,98 \\
\hline 2.1. Colaboración y coordinación & 5 & 1,37 \\
\hline 2.2. Desarrollo profesional docente & 12 & 3,30 \\
\hline 2.3. Implicación y participación & 35 & 9,64 \\
\hline 2.4. Liderazgo & 10 & 2,75 \\
\hline 2.5. Mejora clima escolar & 10 & 2,75 \\
\hline 2.5.1. Mejora de la relación docente-docente & 2 & 0,55 \\
\hline $\begin{array}{l}\text { 2.5.2. Mejora de la relación docente- } \\
\text { estudiante }\end{array}$ & 8 & 2,20 \\
\hline 2.6. Motivación & 13 & 3,58 \\
\hline 2.7. Profesor acompañante & 12 & 3,30 \\
\hline 2.8. Satisfacción personal & 30 & 8,26 \\
\hline 3. Respecto a la familia y agentes externos & 8 & 2,20 \\
\hline 3.1. Participación de agentes externos & 6 & 1,65 \\
\hline 3.2. Participación de la familia & 2 & 0,55 \\
\hline 4. Respecto al centro educativo & 25 & 6,88 \\
\hline 4.1. Apoyo equipo directivo & 19 & 5,23 \\
\hline 4.2. Colaboración de todo el centro & 2 & 0,55 \\
\hline 4.3. Disponer de recursos TIC en el centro & 4 & 1,10 \\
\hline 5. Respecto al currículum educativo & 9 & 2,47 \\
\hline 5.1. Integración del proyecto en el currículum & 9 & 2,47 \\
\hline 6. Respecto al proyecto Atocha Solidaria & 4 & 1,10 \\
\hline 6.1. Metodología utilizada & 4 & 1,10 \\
\hline
\end{tabular}

Como vemos en la Tabla 2, la mayor fuente de satisfacción proviene del impacto que ha tenido la experiencia en el alumnado. Por lo general, los docentes son sensibles a la situación que observan en sus estudiantes y su educación. Valoran mejor una actividad 
cuando tiene un mayor alcance para ellos (Pozuelos, 2007). En este sentido, destacan, como un aspecto positivo, la colaboración entre estudiantes y el apoyo más cercano entre compañeros: "Lo interesante de todo esto es la experiencia entre las dos secciones, y también lo que supone trabajar con otros, porque cuando están en grupo, todos se coordinan, quieren la misma nota, los mismos objetivos, y se ponen las pilas" (Entrevista 4). "Aprender a gestionar el trabajo en equipo" (Entrevista 9). "Han conseguido trabajar en equipo, resolver proyectos, relacionarse con gente externa" (Entrevista 12). Estamos viendo como el ABP propicia la colaboración entre el alumnado, la realización de tareas en pequeños y grandes grupos, el intercambio de ideas y la negociación de soluciones. Estas situaciones facilitan una verdadera interacción y relación entre el alumnado.

También perciben esta experiencia como una oportunidad para formar alumnos críticos y sensibles con los problemas que ocurren en la sociedad, capaces de desarrollar valores sociales basados en la solidaridad, el respeto, la justicia social o los derechos humanos, entre otros: "A mí lo que más me gusta de este proyecto es luego la visión que tiene de concienciación a los chicos" (Entrevista 10). "Es un proyecto que tiene un grado alto de civismo, de aprender que no estás solo, que hay organizaciones que no conoces (...) y luego muchas organizaciones que te das cuenta que hay muchas personas voluntarias trabajando con colectivos de prostitutas, de drogadictos, niños con problemas, se ha hecho una labor grande de concienciación..." (Entrevista 7). Consideramos que la democracia se refuerza cuando la escuela procura la formación de ciudadanos como resultado de participar de manera efectiva en las decisiones que les afectan.

Y como no, los docentes estiman con especial transcendencia que los estudiantes han adquirido competencias digitales, dado que han utilizado herramientas de la web $2.0 \mathrm{y}$ aplicaciones para móviles y tablets con las que manejar la información en diferentes formatos (vídeos, audio, presentaciones, texto,... ). Los alumnos han adquirido así a lo largo del trabajo un gran manejo de las tecnologías lo cual les será de gran utilidad para su presente y su futuro: "Han utilizado twitter como red social, el blog, herramientas de dibujo, canva para los carteles, geogebra, códigos QR, por supuesto, todas las google app, y para el tema de las apps, unos han estado con app inventor, otros con varias aplicaciones que son para hacer prototipos de app, se llama mockup (...) en mi asignatura hemos trabajado toda la parte conceptual, pero más que conceptual, las mías son de herramientas, la competencia digital" (Entrevista 1). "Crearon un vídeo de la ONG, que luego tenían que generar el código QR para ponerlo en el Medialab, y que la gente pudiera acceder a esta información en inglés" (Entrevista 11). "Me parece muy positivo sobre todo porque las tecnologías ejercen una fase en la identidad bastante importante de reconocimiento, de difusión, de confianza en uno mismo, tu fijate me ha retwitteado, y se van súper contentos, entonces me parece muy positivo y como dice Ortega y Gasset hay que estar a la altura de los tiempos" (Entrevista 2).

Pero todos estos elementos ganan en profundidad gracias al elevado nivel de compromiso e implicación que declaran los participantes: "Cada uno lleva sus procesos, lo habrás visto en el claustro cada uno tiene sus velocidades, diferentes niveles, pero en general, yo creo que no hay que convencer a nadie de que esto hay que hacerlo, y eso antes había que convencer, antes teníamos discusiones en el claustro" (Entrevista 1). "Yo creo que la implicación ha sido alta, en general sí (...) lo mejor la coordinación de los profesores que hemos estado muy encima, todos los días nos preguntábamos por el grupo de whatsapp qué toca ahora, cómo se hace, nos dábamos ideas, había soporte también dentro de las aulas, para mí eso ha sido muy bueno a nivel docente" (Entrevista 7). "En cuanto al equipo de profesores yo creo que ha habido bastante implicación por la mayoría de los profesores (...) hay un grupo de profesores súper involucrados, creemos mucho en este tipo de trabajo y nos convence, muchos profes que han estado implicados, Silvia, Patri, Ana con el tema del montaje" (Entrevista 9). 
En definitiva, la valoración de la experiencia que realiza el profesorado es altamente positiva. El grado de satisfacción expresado es alto y han superado el nivel de expectativas que tenían sobre los estudiantes: "Ha superado las expectativas porque han hecho cosas súper chulas, creo que incluso si el próximo año se repite, hay cosas que se les puede sacar más jugo" (Entrevista 1). "Para mí el proyecto ha merecido la pena, porque veo que es una forma que tu cambias" (Entrevista 10). "Una experiencia muy positiva, porque tu ten en cuenta, que hemos pasado de trabajar los textos periodísticos desde la distancia, que tu traes unos ejemplos que trabajan en clase a haber hecho un taller único y exclusivo de entrevistas, a haberse documentado sobre el tema, de manera que las preguntas que se han hecho han sido interesantísimas; a ver cuándo las cuelgo en el blog para que tengas acceso, y hay realmente entrevistas fabulosas, fabulosas porque realmente ves que los chicos conocen el tema" (Entrevista 13).

A su vez, que la experiencia haya contado con un asesoramiento continuado por parte de la coordinadora del proyecto, durante el proceso, lo interpretan como una medida imprescindible: "Evidentemente la voz cantante la ha llevado Charo, sin ella no hubiese sido posible sacar adelante esto (...) Charo que es la que planteaba un poco las jornadas, el guion, la planificación del trabajo" (Entrevista 9). "Tenía que preguntar a Charo, a ti también te preguntaba" (Entrevista 11). "Charo hacía unos cuadrantes con profesores" (Entrevista 8). "Hay profesores como Charo, que nos ayuda a los que sabemos menos" (Entrevista 3). Lo que nos lleva a señalar la importancia de esta figura en el proyecto, que trata de orientar, dinamizar y promover decisiones deliberadas de forma conjunta.

Otros beneficios nos conducen a la dirección del centro educativo. Desde este apartado se hace referencia al apoyo constante del equipo directivo, que sin ellos hubiese sido imposible realizar la experiencia: “... un 10 al equipo de dirección, creo que sin ella no se podría hacer..." (Entrevista 1). "Estoy admiradísimo, sinceramente, el equipo directivo la facilidad que ha dado y Ana como miembro del equipo directivo las grandes facilidades que ha dado tanto de horario, flexibilidad y demás" (Entrevista 12). "La dirección titular ahora esto lo apoya, yo creo que lo respalda, el director actual creo que esta forma de trabajar le gusta, se ha interesado también es bueno que viniera el día de la exposición final, y eso demuestra que nos apoya" (Entrevista 6).

\section{Discusión y conclusiones}

Los resultados obtenidos muestran una valoración muy positiva por parte de la mayoría de los docentes sobre el proyecto Atocha Solidaria, en concreto la estrategia adoptada basada en Design for change, en base a su experiencia escolar; y quedan expectantes ante futuras experiencias de carácter similar. La forma de trabajo es uno de los aspectos que los docentes valoran de manera positiva, destacan que los estudiantes han aprendido y desarrollado diversas destrezas y habilidades, especialmente el trabajo en equipo y el uso de herramientas digitales. $Y$ es que la colaboración entre los estudiantes tiene un enorme potencial para enriquecer mutuamente las experiencias de aprendizaje de los participantes (Hunter y Botchwey, 2017). Los alumnos fueron organizados en grupos de cuatro o cinco integrantes con diferentes capacidades. Consideramos que tales agrupaciones heterogéneas permiten poner en práctica múltiples perspectivas y diversas habilidades, mejorando la calidad del trabajo del proyecto y creando nuevas avenidas para la especialización individual y la tutoría entre pares. Larmer, Mergendoleer y Boss (2015) descubrieron que la colaboración era una gran ventaja en el $A B P$, ya que los estudiantes mejoraban sus habilidades de cooperación y comunicación, aprendían activamente unos de 
otros y asumían más responsabilidades. A su vez, el trabajo en equipo puede favorecer un mejor ambiente de trabajo y clima en el aula (Bell, 2010).

Además de la colaboración, los docentes valoran el papel del proyecto en la formación de valores personales y sociales en los estudiantes, de participación activa y crítica en la sociedad. De hecho, los alumnos han contactado y colaborado estrechamente con diferentes ONG y han utilizado su conocimiento para mejorar la labor de estas organizaciones. Este servicio se ha convertido en una experiencia de aprendizaje de gran valor que les ha proporcionado diversos conocimientos y valores. De este modo el proyecto ha contribuido a una educación para la ciudadanía activa y responsable, los estudiantes han descubierto el sentido y la utilidad de lo que aprenden, implicándose en dicha acción y mejorando su actitud y motivación para el aprendizaje (Opazo, Ramírez, García-Peinado y Lorite, 2015)

Las tecnologías también han sido un elemento esencial del proceso y han apoyado de forma especial la realización de este proyecto de aprendizaje. Han representado una oportunidad para buscar información, contrastarla, compartirla y difundirla en internet. Siguiendo a Cope (2003), la tecnología tenía el potencial de generar aprendizajes de calidad porque proporcionaba los medios para obtener retroalimentación de manera apropiada y oportuna; apoyó el proceso de investigación y la naturaleza asíncrona de la tecnología les dio a los estudiantes un sentido de responsabilidad sobre su propio aprendizaje. De este modo, las TIC juegan un papel muy importante dentro de los discursos sobre ABP, ya que pueden contribuir al desarrollo de procesos de aprendizaje en donde los saberes se ejerciten buscando solucionar situaciones funcionales, complejas y cotidianas (Roegiers, 2004).

Durante el proyecto, también se pone de manifiesto la mejora de las relaciones entre iguales en el aula, pero a su vez, la mejora de las relaciones docente-discente promoviéndose una relación afable y un cambio de actitudes, que generan en el alumnado una mejor predisposición hacia el aprendizaje. EI ABP busca romper con la relación clásica profesor-alumno, en la que el docente solo tiene el rol de enseñar y el discente el de aprender, para pasar a establecer una relación basada en la comunicación, donde ambos actores tienen poder en la toma de decisiones (Balongo y Mérida, 2016).

Para el desarrollo del proyecto ha sido también fundamental la implicación de todo el profesorado, y el propio funcionamiento del grupo de docentes que ha participado, donde se ha generado un clima de confianza entre ellos, lo cual ha facilitado el trabajo, la discusión y la toma de decisiones. Todos son conscientes de que individualmente hubiese sido imposible llevar a cabo la experiencia. Están muy satisfechos con el grado de cohesión del grupo y con el crecimiento profesional y personal que ha supuesto para ellos (Pozuelos, 2007).

Se señala a su vez la motivación que esta metodología supone para el profesorado al romper con la rutina en el tratamiento de los temas. $Y$ es que el éxito del ABP depende también de la motivación y el apoyo con que cuenten los docentes en su tarea de facilitar la investigación. Por último, la formación y el desarrollo profesional que han experimentado satisfacen de forma indiscutible al profesorado implicado en este estudio. El ABP irrumpe como un proceso que le involucra y le ayuda a mejorar sus competencias profesionales, que le permite atender la formación con entusiasmo y desde otra perspectiva (Habok y Navy, 2016; Lacueva, 2016). Los docentes aprenden colaborando con sus colegas, presentando el ABP en el aula y reflexionando sobre sus experiencias (Krajcik y Blumenfeld, 2006). Existen redes colaborativas en línea de apoyo al profesorado en el desarrollo de sus prácticas 
docentes sobre los métodos de ABP. En estos entornos los docentes comparten sus ideas, reciben comentarios e interactúan con otras aulas de $A B P$ a través de la red.

\section{Agradecimientos}

Esta investigación fue posible gracias a la estrecha colaboración del centro educativo de Madrid, especialmente del equipo directivo, los profesores y estudiantes de primero de Bachillerato y la principal coordinadora del proyecto Atocha Solidaria.

También ha sido posible gracias a la financiación de una ayuda predoctoral de la Junta de Castilla y León, cofinanciada por el Fondo Social Europeo (Orden EDU/1083/2013, de 27 de diciembre).

\section{Referencias}

Badía, A. y García, C. (2006). Incorporación de las TIC en la enseñanza y el aprendizaje basados en la elaboración colaborativa de proyectos. RUSC, Revista de Universidad y Sociedad del Conocimiento, 3(2), 42-53.

Balongo, E. y Mérida, R. (2016). El clima de aula en los proyectos de trabajo. Crear ambientes de aprendizaje para incluir la diversidad infantil. Perfiles educativos, 38(152), 146-162.

Barak, M. y Dori, Y. (2004). Enhancing undergraduate students' chemistry understanding through project based learning in an IT environment. Science Education, 89(1), 117139.

Bas, G. (2011). Investigating the effects of Project based learning on students'academic achievement and attitudes towards English lessons. TOJNED : The Online Journal Of New Horizons In Education, 1(4), 1-15.

Beaugrande, R. y Dressler, W. (1997). Introducción a la linguística del texto. Barcelona: Ariel.

Bell, S. (2010). Project based learning for the 21st century: skills for the future. The Clearing House, 83, 39-43.

Cámara, A., Díaz, E. y Ortega, J. (2017). Aprendizaje-servicio en la Universidad: ayudando a la escuela a atender a la diversidad a través de las TIC. Bordón, 69(3), 1-15. doi: 10.13042/Bordon.2017.51320

Cope, C. (2003). A framework for using learning technologies in higher education to enhance the quality of students' learning outcomes. En G. Crisp, D. Thiele, I. Scholten, S. Barker and J. Baron (Eds.), Interact, Integrate, Impact: Proceedings of the 2oth Annual Conference of the Australian Society for Computers in Learning in Tertiary Education (pp. 134-141). Australia: Adelaide.

Escofet, A., Folgueiras, P., Luna, E. y Palou, B. (2016). Elaboration and Validation of a Questionnaire for the Evaluation of Service-Learning Projects. Revista mexicana de investigación educativa, 21(70), 929-949.

García-Valcárcel, A. (coord.) (2015). Proyectos de trabajo colaborativo con TIC. Madrid: Síntesis. 
García-Varcálcel, A. y Basilotta, V. (2017). Aprendizaje basado en proyectos (ABP): evaluación desde la perspectiva de alumnos de Educación Primaria. Revista de Investigación Educativa, 35(1), 113-131. doi:http://dx.doi.org/10.6018/rie.35.1.246811

García-Valcárcel, A., Basilotta, V. y López, C. (2014). Las TIC en el aprendizaje colaborativo en el aula de primaria y secundaria. Comunicar, 42(21), 65-74.

Habok, A. y Nagy, J. (2016). In service teachers' perceptions of project based learning. SpringerPlus, 5(83), 1-14. doi: 10.1186/s40064-016-1725-4

Hunter, P. E. y Botchwey, N. D. (2017). Partnerships in Learning: A Collaborative Project between Higher Education Students and Elementary School Students. Innovative Higher Education, 42(1), 77-90.

Krajcik, J. y Blumenfeld, P. (2006). Project based learning. En R. Keith (Ed.), The Cambridge Handbook of the Learning Sciences (pp.317-334). EEUU: Cambridge University.

Lacueva, A. (2016). La educación por proyectos abre caminos hacia una escuela más viva y profunda. Red. Revista de evaluación para docentes y directivos, 3, 68-77.

Lacueva, A. Imbernon, F. y Llobera, R. (2003). Enseñando por proyectos en la escuela: la clase de Laura Castell. Revista de Educación, 332, 131-148.

Larmer, J., Mergendoller, J. y Boss, S. (2015). Setting the Standard for Project Based Learning: A Proven Approach to Rigorous Classroom Instruction. Novato, California: Buck Institute for Education.

Mayor, D. y Rodríguez, D. (2016). Aprendizaje-servicio y práctica docente: una relación para el cambio educativo. Revista de Investigación Educativa, 34(2), 535-552. doi: http://dx.doi.org/10.6018/rie.34.2.231401

Opazo, H., Ramírez, C., García-Peinado, R. y Lorite, M. (2015). La ética en el aprendizajeservicio (ApS): Un meta-análisis a partir de Education Resources Information Center (ERIC). Profesorado, revista de currículum y formación del profesorado, 19(1), 145-175.

Pozuelos, F. J. (2007). Trabajo por proyectos: descripción, investigación y experiencias. Morón (Sevila): Ediciones MCEP.

Puig, J., Gijón, M., Martín, X. y Rubio, L. (2011). Aprendizaje-servicio y Educación para la Ciudadanía. Revista de Educación, número extraordinario, 45-67.

Railsback, J. (2002). Project-based instruction: Creating excitement for learning. Portland, Oregon: Northwest Regional Educational Laboratory.

Roegiers, X. (2004). Una pedagogía de la integración. Bruselas: Deboeck Universidad.

Stenhouse, L. (1991). Métodos de estudio de casos. En T. Husan y T.N. Postlethwaite (Eds.), Enciclopedia Internacional de la Educación (pp. 3911-3916). Madrid: Vicens Vives.

Vázquez, S., Liesa, M. y Lozano, A. (2017). Recreos Cooperativos e Inclusivos a través de la metodología de Aprendizaje-Servicio. Revista Electrónica Interuniversitaria de Formación del Profesorado, 20(1), 173-185. doi: http://dx.doi.org/10.6018/reifop.20.1.213181 